\title{
USE OF FREE AND OPEN-SOURCE SOFTWARE APPS PROVIDE MANY BENEFITS FOR COLLEGE STUDENTS
}

\author{
Jeffrey A. Nowak, Professor, Purdue University Fort Wayne, janowak@purdue.edu
}

\section{Abstract, Concept, and Research Questions}

Free and Open-Source software apps along with free Linux-based distributions of operating systems have developed at a blistering pace over the past decade. Today more than ever before, it is possible for students to download and use free software to complete a plethora of complex tasks related to educational pursuits. This paper considers the impact of allowing students to explore the use of more than a dozen cross-platform, free, and open-source software apps to complete STEM-related tasks in a University preservice education course. The core cross-platform apps install in Windows 10, macOS, and various Linux distributed operating systems. In addition, they are supported to work in Chrome OS via the Crostini project.

\section{Methodology and Findings}

The apps used in this project are free, open-source, and widely available to anyone via download from their respective websites. Google Search was the primary browser-based search tool employed in the initial search for apps that might assist in the completion of assignments related to an introductory STEM preservice teacher course at the University. The various tasks needed to complete the assignments included converting digital files used in science skills learning labs and creating lesson plan activities into web-friendly formats for online sharing and virtual learning. Since course content includes introducing students to foundational science and mathematics-based conventions and technology skills, apps that would produce files best-suited for online sharing were sought and vetted over a two-year time span. In that time, given the ever-evolving landscape of technology, some cross-platform apps held periods of time where they worked better than others on different operating system platforms. Most of the apps were typically upgraded and released in new versions, revised to work with newer operating system releases within 2 to 6 months.

The tasks sought to be met by the apps included: basic word processing, advanced file display, and encoding and conversion for cross-platform online sharing. Partially driven by modern cloud-based file sharing and end-user browser capabilities, JPG, PNG, MP3, MP4, PDF, and TXT file formats were shared online among peers, while word processing apps capable of reading DOCX formatted text were used to create PDF's with active hyperlinks to embedded and streamed media in these formats. This was thought to be the best approach given the wide range of digital devices future parents and students of the preservice teachers may use. Using formats supported via streaming directly within modern browsers helps to ensure documents are viewed by recipients as intended without a need for additional software.

\section{Referenced Software}

Axcore. axcore/tartube. GitHub. https://github.com/axcore/tartube.

Crook, J. (2020, October 24). Home. Audacity ®. https://www.audacityteam.org/.

diagrams.net - free flowchart maker and diagrams online. Draw.io. https://app.diagrams.net/.

fre:ac. freac.org. https://www.freac.org/.

GIMP. https://www.gimp.org/.

A hackable text editor for the 21st Century. Atom. (2021). https://atom.io/.

HandBrake The open-source video transcoder. HandBrake RSS. https://handbrake.fr/.

OpenOffice Evolution. LibreOffice. https:/www.libreoffice.org/.

PDF SAM Basic. pdfsam.org. https://pdfsam.org/.

Shotcut. https://shotcut.org/.

Thibaud.be. XnView Software XnViewMP. XnView Software. https://www.xnview.com/en/xnviewmp/.

VideoLAN. VLC media player. VideoLAN. https://www.videolan.org/vlc/index.html. 